\title{
Green Tea Treatment Attenuates Oxidative Damage and Neuromotor Deficit Induced by an Experimental Model of Intracerebral Hemorrhage in Rats
}

\author{
${ }^{1}$ Mauren Assis Souza, ${ }^{1}$ Caroline Dalla Colletta Altermann, \\ ${ }^{1}$ Alexandre dos Santos Martins, ${ }^{1,2}$ Priscila Marques Sosa, \\ ${ }^{3}$ Cristiano Chiapinotto Spiazzi, ${ }^{3}$ Francieli Weber Santos and ${ }^{1,2}$ Pamela Billig Mello-Carpes \\ IPhysiology Research Group, Stress, Memory and Behavior Lab, \\ Federal University of Pampa, BR 472, Km 592, Po Box 118, Zip code 97500-970, Uruguaiana/RS/Brazil \\ ${ }^{2}$ Graduate Program in Biological Sciences, Physiology, Federal University of Rio Grande do Sul, Porto Alegre/RS/Brazil \\ ${ }^{3}$ BIOTECH, Federal University of Pampa (UNIPAMPA), 97.500-970, Uruguaiana, RS, Brazil
}

Corresponding Author:

Pamela Mello-Carpes

Physiology Research Group,

Stress, Memory and Behavior

Lab, Federal University of

Pampa, BR 472, Km 592, Po

Box 118, Zip code 97500-970,

Uruguaiana/RS/Brazil

Email: pamelacarpes@unipampa.edu.br

\begin{abstract}
The present study shows the neuroprotective effect of green tea supplementation (GT; Camellia sinensis) in a model of Intracerebral Hemorrhage (ICH) in rats. ICH was induced by intra-striatum collagenase infusion in male Wistar rats. GT ( $400 \mathrm{mg} \mathrm{Kg}^{-1}$ ) was administered via gavage for 10 days after ICH. We assessed the effect of GT on neuromotor recovery and oxidative damage. Our results show that ICH causes neuromotor deficits observed by Neurological Deficit Scale (NDS), Open Field (OF) and Rotarod (RR). GT treatment attenuates this deficit on day 3 for NDS and OF and on day 7 for RR. Also, ICH increases Reactive Oxygen Species (ROS) production and lipid peroxidation, what is not observed in ICH + GT group. Currently, there are no effective pharmacologic or non-pharmacologic neuroprotective treatments for ICH. Nutritional interventions that help on its outcome are important, since they are generally accessible and have few side effects.
\end{abstract}

Keywords: Hemorrhagic Stroke, Green Tea, Nutritional Intervention, Neuroprotection, Neurological Deficit, Oxidative Imbalance

\section{Introduction}

Hemorrhagic stroke caused by spontaneous ICH represents 15 to $20 \%$ of all strokes (Flower and Smith, 2011). ICH is the more severe type of stroke and has serious development (Hwang et al., 2011). Most patients either die or are left with significant neurological morbidity (Flower and Smith, 2011). Moreover, the incidence of ICH is expected to grow, considering the increase on life expectancy of population.

The primary damage caused by ICH starts soon after the bleeding onset and is mainly due to hematoma formation, which compresses the surrounding brain tissue, thus destroying it (Xi et al., 2006). The progressive brain tissue deterioration suggests that secondary brain damage after ICH plays a fundamental role in neurological impairment (Babu et al., 2012), even without any signs of hematoma expansion.

Neurochemical alterations induced by secondary events cascade after ICH have not been well delineated, but they may represent important therapeutic targets to attenuate further brain damage. As a result of hemorrhage, hemoglobin and its breakdown products (iron, biliverdin and carbon monoxide) get in touch with brain parenchyma, activating cytotoxic, oxidative and inflammatory cascades (Xi et al., 2006; Aronowski and Zhao, 2011; Hu et al., 2016). Iron and iron-related compounds, including hemoglobin, increase hydroxyl radical production and oxidation of lipids (Sadrzadeh and Eaton, 1988), which expose the brain to higher levels of ROS. There is increasing evidence that oxidative stress contributes to ICH-induced secondary brain injury via generation of ROS (Hu et al., 2016). Despite the advances in research and care, very limited options of treatments are available and the recovery of patients with $\mathrm{ICH}$ is still poor. There is a compelling need for more options of therapeutic and adjuvant therapies to aid this critical population. Potential nutritional interventions targeting secondary brain injury are arousing a great deal of interest, especially those with an antioxidant component. 
Green tea (Camellia sinensis) has been reckoned as a possible source of antioxidants available through diet (Wu et al., 2012; Xu et al., 2010). Catechins, a group of flavonoids, constitute around $30-45 \%$ of the solid GT extract (reviewed in (Mak, 2012)). The beneficial effects of tea consumption are probably due to its bioactive components (catechins and their derivatives), demonstrated to act directly as radical scavengers and to exert indirect antioxidant effects through activation of transcription factors and phase II antioxidant defense, thus modulating the cellular redox state (see review: Mandel et al., (2006). The Epigallocatechin Gallate (EGCG) is a major component of GT and the administration of both GT extract and EGCG has shown to be a neuroprotective compound in experimental models of ischemic stroke, protecting the brain against oxidative stress, neuromotor deficit and lesion volume in both rats and mice (Wu et al., 2012; Chang et al., 2014; Shah et al., 2010; Schimidt et al., 2014).

Although ischemia and ICH share many secondary events cascade, fundamental physiopathological differences remain (Shah et al., 2010).

Thus, it is important to test potential treatments in $\mathrm{ICH}$ models instead of rely on research findings in ischemia models. To study the effects of a popular drink such as GT could be a useful tool for the treatment of $\mathrm{ICH}$. Besides, this is a non-invasive low cost nutritional intervention. Therefore, our aim is to investigate whether green tea treatment can protect against oxidative damage and neuromotor deficit induced by $\mathrm{ICH}$ in rats.

\section{Materials and Methods}

\section{Animals and Reagents}

All procedures followed the "Principles of laboratory animal care" (NIH publication No. 80-23, revised 1996) and the guidelines established by the Institutional Animal Care and Use Committee of the Local Institution (Approval n. 014/2014). We used 41 male Wistar rats (250-300 g, 3 months old) randomly assigned to 4 groups with blinded assessment. Rats were grouped 4 per cage and maintained under controlled environmental conditions $(12 \mathrm{~h}$ light/12 h dark cycle at temperature $23 \pm 2{ }^{\circ} \mathrm{C}$ and humidity $50 \pm 10 \%$ ) with food and water ad libitum.

Green tea was purchased from Madrugada Co. All the other drugs were purchased from Sigma-Aldrich.

\section{Experimental Procedures}

\section{Surgery and Experimental Groups}

Surgical procedures were performed aseptically. Rats were anesthetized with ketamine and xylazine (i.p., 75 and $10 \mathrm{mg} \mathrm{kg}^{-1}$, respectively). Body temperature was maintained at $37^{\circ} \mathrm{C}$ during anesthesia with a heating pad. After placing the animals in a stereotaxic frame a hole was drilled $3.5 \mathrm{~mm}$ right and $0.5 \mathrm{~mm}$ anterior to Bregma. A 26-gauge needle (Hamilton syringe; Hamilton, Reno, $\mathrm{NV}$, USA) was inserted $6.5 \mathrm{~mm}$ unilaterally into the right striatum to infuse $1 \mu \mathrm{L}$ of sterile saline, containing $0.2 \mathrm{U}$ bacterial collagenase (Type IV) in the case of ICH groups, over $5 \mathrm{~min}$. The needle was left in place for 5 min and then slowly removed. The hole was sealed with a metal screw, clips were used to close the wound and lidocaine was used.

Animals were randomly divided into four groups: Sham $(\mathrm{n}=10)$; ICH $(\mathrm{n}=10)$; Sham + GT $(\mathrm{n}=11)$; ICH + GT $(\mathrm{n}=10)$.

\section{Green Tea Supplementation}

Animals treated with GT had the first oral administration by gavage (400 $\mathrm{mg} \mathrm{Kg}^{-1}$ ) (Xu et al., 2010) four hours after surgery and for the following 10 days (Chang et al., 2014). GT infusion of $400 \mathrm{mg} \mathrm{kg}$ per day for rats is equivalent to $400 \mathrm{~mL} \mathrm{day}^{-1}$ for humans (Xu et al., 2010).

Green tea infusion was freshly prepared daily and administered at ambient temperature. Control groups received the same volume of tap water. Green tea samples from Madrugada Co. used in this study were purchased from standard markets and analyzed by High Performance Liquid Chromatography (HPLC), which ensured the presence of epicatechin (concentration of 1.3 $\left.\mathrm{mg} \mathrm{mL}^{-1}\right)$, epigalocatechin gallate EGCG $\left(3.7 \mathrm{mg} \mathrm{mL}^{-1}\right)$ and Epicatechin Gallate (ECG) $\left(1.8 \mathrm{mg} \mathrm{mL}^{-1}\right)$.

\section{Behavioral Assessment}

All animals were subjected to behavioral assessment followed by biochemical analyses at 11-day survival (Fig. 1).

\section{Neurological Deficit Scale (NDS)}

This battery of tests sensitive to striatal damage (Del Bigio et al., 1996) was used on post-surgery days 1, 3 and 7 (vs. days before surgery). Briefly, rats were assessed on spontaneous circling, hind limb retraction, bilateral forepaw grasp, contralateral forelimb flexion and beam walking. A maximum score of 14 denotes greatest neurological impairment.

\section{Open Field}

The open field test was carried out in a wooden box $(50 \times 60 \times 60 \mathrm{~cm})$ divided into 16 regular square areas. Each rat was set in the center of the box at the beginning of the session and was allowed to explore the arena for 5 min. Locomotion (number of squares crossed with the four paws), was assessed. About $70 \%$ ethanol was used to clean the arena between each rat. 


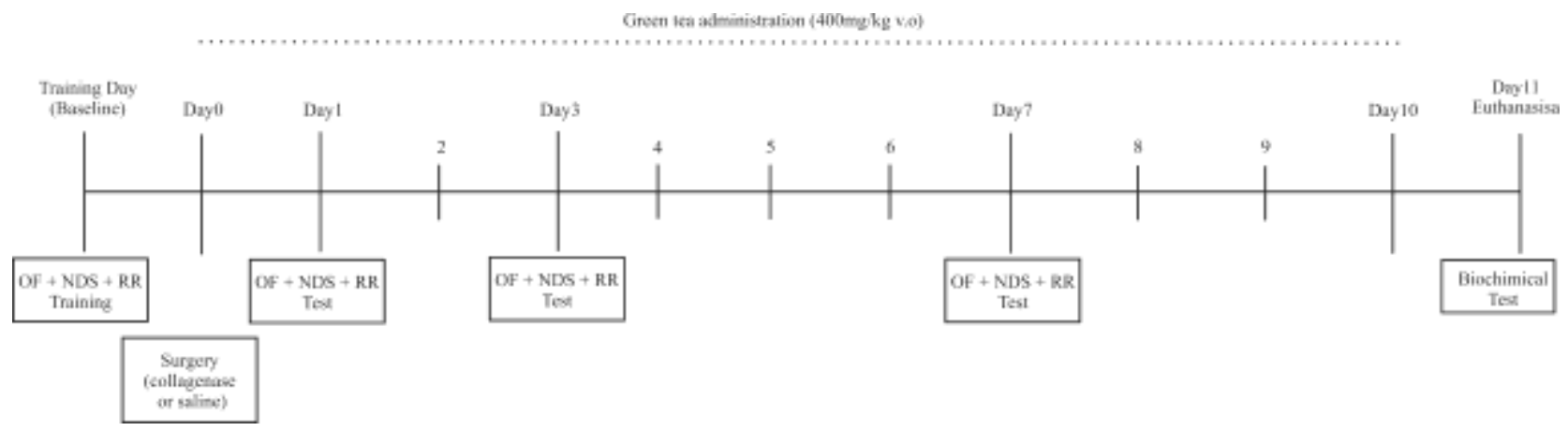

Fig. 1. Representation of experimental design. One day before surgery, animals were trained in Open Field task (OF), Neurological Deficit Scale (NDS) and Rota Rod (RR) for baseline values. At day 0 the surgery procedure was performed to induce hemorrhage injury (collagenase) or sham injury (saline). Green tea treatment started four hours after injury and lasted 10 days. The animals were tested on OF, NDS and RR at day 1, 3 and 7 after surgery

\section{Rotarod}

Rotarod is a test that assesses motor coordination, balance and muscle strength. The apparatus consists of a cylinder with a diameter of $7.62 \mathrm{~cm}$; suspended $20 \mathrm{~cm}$ from the device surface, run by a motor that keeps a constant speed.

Before the training session, rats were habituated to stay on the stationary drum for $3 \mathrm{~min}$. Habituation was repeated every day (days 1, 3 and 7) for 1 min just before the testing session. On the training day, the speed used was of 16 rotations per minute and on testing days, it was of 25 rotations per minute.

Rats were placed on the cylinder and the first fall was timed to a maximum of $360 \mathrm{sec}$ as well as the number of falls up to five times in one session (Shiotsuki et al., 2010; Stroobants et al., 2013).

\section{Biochemical Assays}

\section{Tissue Preparation}

Rats of all groups were euthanized $24 \mathrm{~h}$ after the last GT administration. Their brains were removed and ipisilateral striatum was quickly dissected out and homogenized in $50 \mathrm{mM}$ TrisHCl, $\mathrm{pH} 7.4,(1 / 10, \mathrm{w} / \mathrm{v})$. Afterwards, samples were centrifuged at $2400 \mathrm{~g}$ for 10 min and supernatants (S1) were used for assay.

\section{Reactive Oxygen Species (ROS)}

Spectrofluorimetric method using 2,7dichlorofluorescein diacetate (DCFH-DA) as a probe was used to evaluate ROS content (Ali et al., 1992). The sample (S1) was incubated in the dark with $5 \mu \mathrm{L}$ DCFHDA (1 mM). Detection of intracellular ROS was given by DCHF-DA oxidation to fluorescent dichlorofluorescein (DCF) The formation of the oxidized fluorescent derivative (DCF), measured by DCF fluorescence intensity, was recorded at $520 \mathrm{~nm}(480 \mathrm{~nm}$ excitation) $30 \mathrm{~min}$ after the addition of DCFH-DA to the medium. Results were expressed as \% of control of AU (arbitrary units).

\section{Detection of TBARS Level}

Lipoperoxidation was assessed through the Thiobarbituric Acid Reactive Substance (TBARS) test (Ohkawa et al., 1979). One aliquot of S1 was incubated with a $0.8 \%$ thiobarbituric acid solution, acetic acid buffer ( $\mathrm{pH} 3.2$ ) and sodium dodecyl sulfate solution $(8 \%)$ at $95^{\circ} \mathrm{C}$ for $2 \mathrm{~h}$. Color reaction was measured at 532 $\mathrm{nm}$ and results were expressed as nmol of Malondialdehyde (MDA) per mg of protein.

\section{Glutathione (GSH)}

GSH levels were fluorometrically determined (Hissin and Hilf, 1976). An aliquot of homogenized sample was mixed $(1: 1)$ with perchloric acid $\left(\mathrm{HClO}_{4}\right)$ and centrifuged at $3000 \mathrm{~g}$ for $10 \mathrm{~min}$. This mixture was centrifugated the protein pellet was discarded and free thiols (SH) groups were determined in the clear supernatant. An aliquot of supernatant was incubated with orto-phthaladehyde and fluorescence was measured at excitation of $350 \mathrm{~nm}$ and emission of $420 \mathrm{~nm}$. Results were normalized by $\mathrm{mg}$ of protein and expressed as percent of control.

\section{Glutathione Peroxidase (GPx) Activity}

GPx activity was analyzed spectrophotometrically by the method of Paglia and Valentine (1967). GPx analysis was made by adding GSH, GR, NADPH and a peroxide to start the reaction, monitored at $340 \mathrm{~nm}$ as NADPH is converted to $\mathrm{NADP}^{+}$.

\section{Protein Determination}

Protein content was measured colorimetrically by the method of (Bradford, 1976) using bovine serum albumin $\left(1 \mathrm{mg} \mathrm{mL}^{-1}\right)$ as standard.

\section{Statistical Analysis}

Results are presented as mean \pm Standard Error of the Mean (SEM). Results were analyzed by Two-way ANOVA with Bonferroni post-hoc test when appropriate. 


\section{Results}

\section{Behavioral Assessment}

Green tea effects were assessed in NDS, OF and RR behavioral tasks. Figure $2 \mathrm{~A}$ shows the effect of a 10-day GT administration (400 $\left.\mathrm{mg} \quad \mathrm{Kg}^{-1}\right)$ on neuromotor deficit induced by ICH assessed in NDS. Two-way ANOVA showed that $\mathrm{ICH}$ induced neuromotor deficit on days $1(\mathrm{~F}(1,37)=43.61 ; \mathrm{P}=$ $0.0001), 3(\mathrm{~F}(1,37)=26.48 ; \mathrm{P}<0.0001)$ and $7(\mathrm{~F}(1,37)$ $=11.42 ; \mathrm{P}=0.001)$ when compared to sham-operated rats. Besides, GT treatment attenuates this impairment on day $3(\mathrm{~F}(1,37)=13.56 ; \mathrm{P}=0.0007)$.

For locomotor activity assessed in $\mathrm{OF}$ the $\mathrm{ICH}$ group presented locomotor disability on days $1(\mathrm{~F}(1,37)=$ 43.95; $\mathrm{P}<0.0001)$ and $3(\mathrm{~F}(1,37)=11.40 ; \mathrm{P}=0.0017)$ after surgery) (Fig. 2B). Besides, the treatment with GT did not change this effect on day $1(\mathrm{~F}(1,37)=0.58$; $\mathrm{P}>0.05)$ though on day $3 \mathrm{GT}$ reverted the locomotion deficit $(\mathrm{F}(1,37)=4.78 ; \mathrm{P}<0.05)$.

We also used the RR test to assess equilibrium and coordination. Figure 3 shows that motor deficit was induced by $\mathrm{ICH}$. This was characterized by the lower latency to first fall (Fig. 3A) on days $1(\mathrm{~F}(1,37)=48.61$; $\mathrm{P}<0.0001), 3(\mathrm{~F}(1,37)=10.55 ; \mathrm{P}=0.0025)$ and 7 $(\mathrm{F}(1,37)=22,7 ; \mathrm{P}<0.0001)$; and an increase on number of falls (Fig. 3B) on all days tested $(\mathrm{F}(1,37)=23.99$; $\mathrm{P}<0.0001$ for day $01 ; \mathrm{F}(1,37)=19.33 ; \mathrm{P}=0.001$ for day $3 ; \mathrm{F}(1,37)=20,39 ; \mathrm{P}<0.0001$ for day 7$)$ in the $\mathrm{ICH}$ group. Green tea treatment was able to revert this motor impairment only on day 7 for both latency to first fall $(\mathrm{F}(1,37)=7.15 ; \mathrm{P}=0.011)$ and number of falls $(\mathrm{F}(1,37)$ $=6.65 ; \mathrm{P}=0.014)$.

\section{Biochemical Assays}

To assess the effect of GT treatment on oxidative imbalance we considered ROS production (Fig. 4A), levels of lipid peroxidation (Fig. 4B), levels of GSH (Fig. 4C) and GPx activity (Fig. 4D). We observed that ICH induced the increase on ROS production $(\mathrm{F}(1,37)$ $=8,91 ; \mathrm{P}=0.005)$ and lipid peroxidation $(\mathrm{F}(1,37)=$ 14.05; $\mathrm{P}=0.0006)$ when compared to sham-operated rats. There was difference between $\mathrm{ICH}+\mathrm{GT}$-treated group when compared to ICH group in $\operatorname{ROS}(\mathrm{F}(1,37)=$ 5.77; $\mathrm{P}=0.02)$, but not in $\operatorname{TBARS}(\mathrm{F}(1,37)=2.44 \mathrm{P}=$ 0.12). However, TBARS levels were not different between sham and ICH + GT-treated group $(\mathrm{F}(1,37)=$ $0.66 ; \mathrm{P}=0.42)$. For antioxidant markers, we observed there were no differences between groups on GSH levels $(\mathrm{F}(1,37)=0.001 ; \mathrm{P}>0.05)$ and $\mathrm{GPx}$ activity $(\mathrm{F}(1,37)=0.26 ; \mathrm{P}>0.05)$.

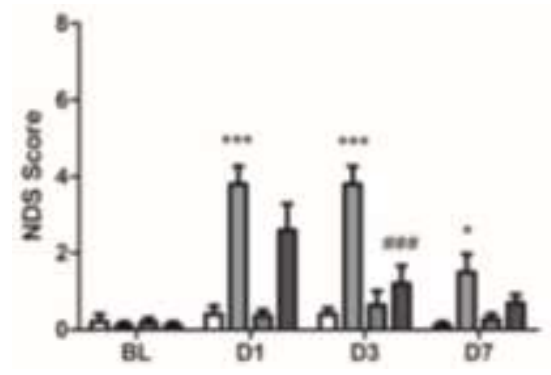

(A)

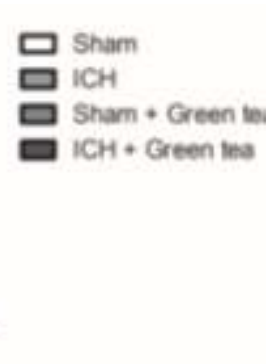

Fig. 2. Effects of green tea administration ( $400 \mathrm{mg} \mathrm{Kg}^{-1}$ p.o) on neuromotor deficit induced by ICH on Neurological Deficit Scale (A) and on locomotor activity (Open Field test) (B) evaluations. Data are presented as the mean \pm S.E.M. Two-Way Anova $* \mathrm{P}<0.05$; $* * \mathrm{P}<0.01$ and $* * * \mathrm{P}<0.001$ compared with vehicle-treated group, $\# \mathrm{P}<0.05$ and \#\# $\mathrm{P}<0.001$ compared with ICH group $(\mathrm{n}=10-11)$

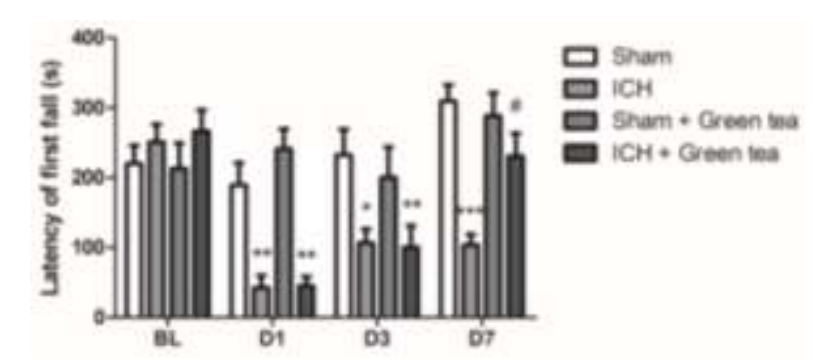

(A)

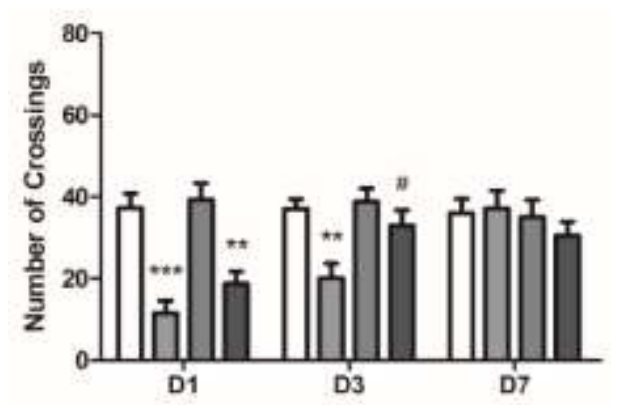

(B) 


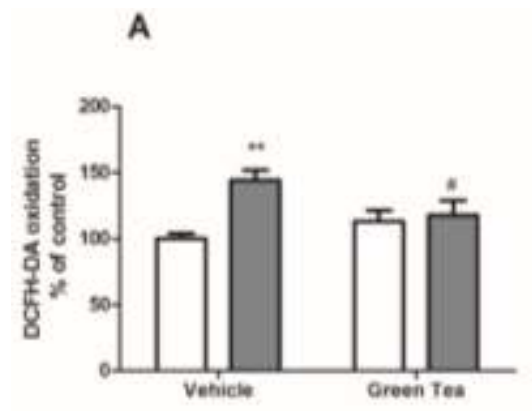

B
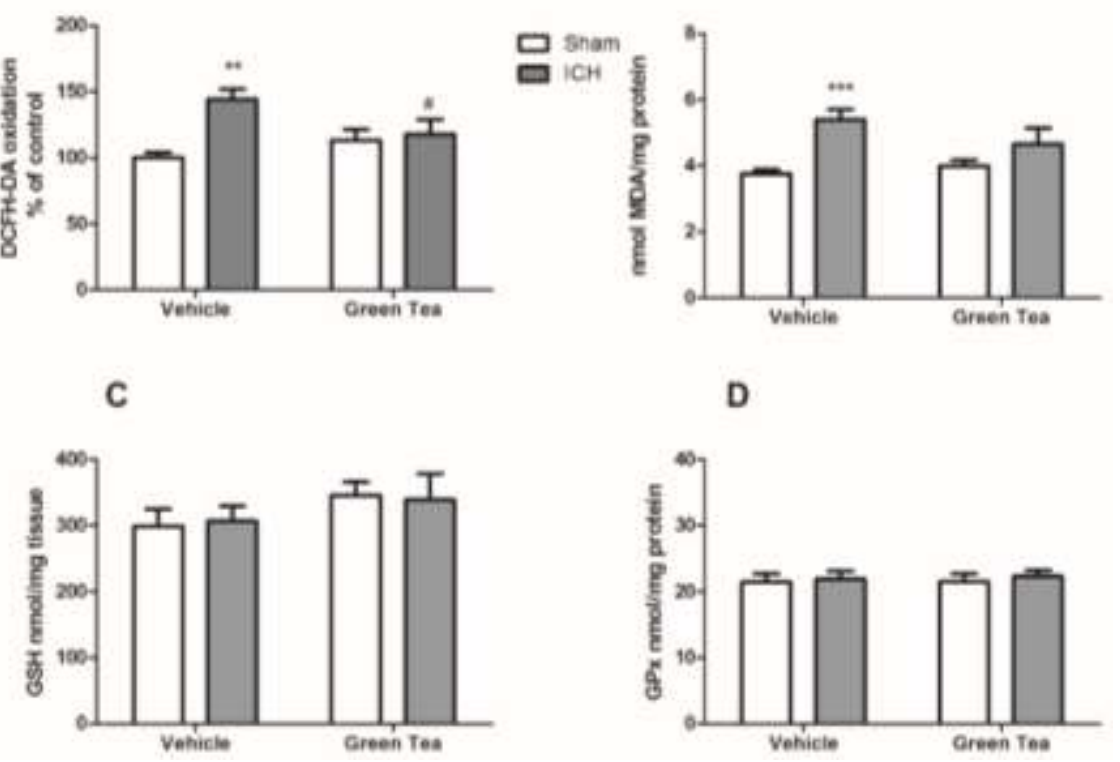

D

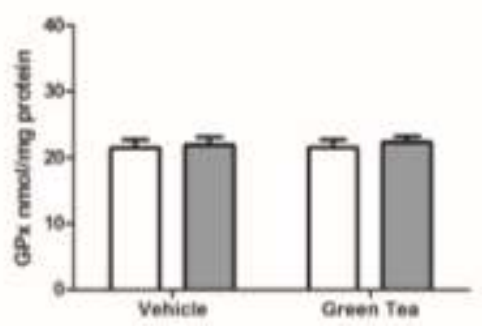

Fig. 4. Effects of green tea administration ( $400 \mathrm{mg} \mathrm{Kg}^{-1}$ p.o) on oxidative status after ICH. (A) Effect of green tea and ICH on ROS production, (B lipid peroxidation, (C) glutathione levels and (D) glutathione peroxidase activity. Data are presented as the mean \pm S.E.M. Two-Way Anova $* * \mathrm{P}<0.01$ and $* * * \mathrm{P}<0.001$ compared with vehicle-treated group, \#P<0.05 compared with $\mathrm{ICH}$ group $(\mathrm{n}=10-11)$

\section{Discussion}

It has been reported that long term green tea consumption is beneficial to human health because the leaves are a source of natural compounds with biological and pharmacological activities (Mandel et al., 2006). Indeed, habitual consumption is associated with a reduced risk of ischemic and hemorrhagic stroke (Tanabe et al., 2008). The beneficial effects of tea consumption are mainly due to bioactive components, catechins polyphenols and their derivatives, which have shown to act directly as radical scavengers and to exert indirect antioxidant effects through activation of transcription factors and antioxidant bioactive components, catechins polyphenols and their derivatives, which have shown to act directly as radical scavengers and to exert indirect antioxidant effects through activation of transcription factors and antioxidant enzymes, thus modulating the cellular redox state (see review: (Mandel et al., 2006)).

Despite the information regarding the effects of GT and GT polyphenols on several neurodegenerative disorders (reviewed in (Mak, 2012; Mandel et al., 2006), there is little information in literature about the use of GT to treat hemorrhagic stroke. In this study, using a collagenase model of ICH in rats, we show that GT treatment attenuates neuromotor deficit and striatum oxidative stress.

Green tea treatment starting four hours following $\mathrm{ICH}$ presented different levels of neuroprotection in these motor tests. In NDS, GT treatment shows positive effects only on day 3. On day 7, the ICH group showed spontaneous recovery, therefore it was not possible to assess the benefits of GT. Similarly to NDS, GT presented positive effects only on day 3 in the OF test. Our results are in accordance with the literature, since $\mathrm{Lu}$ et al. (2015) have previously demonstrated that ICH in basal ganglia induced motor deficit but spontaneous recovery occurs along the days. In the RR test, however, GT was effective only on day 7. This difference in motor recuperation and GT effect can be explained by the complexity to perform the motor test and RR is a more difficult task to perform than NDS and OF since it requires balance, grip strength and motor coordination. This set of data shows that GT attenuates neuromotor deficit induced by hemorrhagic stroke, as previously demonstrated with ischemic stroke. It has been previously shown that GT extract and EGCG administration (the major component of green tea) attenuate neuromotor deficit, oxidative stress and lesion volume in an experimental model of focal and global ischemia (Wu et al., 2012; Chang et al., 2014; Shah et al., 2010).

Chang et al. (2014) had previously studied the effect of Epicatechin (EC) on a model of hemorrhagic stroke and shown that EC induces the activation of Nrf2 and protects against oxidative stress, neuromotor deficit and lesion volume in mice (Chang et al., 2014). In our case, we studied the effects of green tea mixture, without isolating any compound, considering that antioxidant 
characteristic of green tea is not related to a specific kind of polyphenol but to the combined activity of diverse antioxidants present in leaves, including phenolic acids and polyphenols (reviewed (Yang and Landau, 2000)). These data are important, considering that isolated catechins are not always available and GT is more accessible. Also, considering the multifactorial nature of $\mathrm{ICH}$, treatments with specific targets could be ineffective. However, a single drug or cocktail of drugs with pluripharmacological properties could be more appropriate to be employed. ICH is the most devastating subtype of stroke with only $\sim 20 \%$ of survivors regaining functional independence (Flower and Smith, 2011), emphasizing the importance to search for practices and effective strategies to treat it. At present there are no effective treatments and patient management is largely supportive.

It is well known that ROS is normal consequence of metabolic reactions, but high ROS levels can induce cellular death (Juranek and Bezek, 2005). Increasing evidence has shown that oxidative stress is implicated in the development of secondary neuronal injury, including cerebral edema, breakdown of the blood-brain barrier (Hu et al., 2016) and might also contribute to the outcome of ICH (Wang et al., 2006). Indeed, after autologous blood injection protein carbonyl formation, a measure of protein oxidation, have been found detected (Hall et al., 2000; Wagner et al., 2002).

In addition, brain edema and neuromotor were detected after intrastriatal infusion of lysed erythrocytes (Wu et al., 2002). Taken together this studies provide compelling evidences that ROS may contribute to ICH secondary brain injury.

In this study we showed that $\mathrm{ICH}$ induces an increase in ROS production and lipid peroxidation in the striatum. We have chosen the striatum because this structure is directly involved in motor control (Groenewegen, 2003) and because of the ICH model used here (see surgery methods). Importantly, green tea treatment avoids the increase of ROS and lipid peroxidation induced by ICH. In this sense, some studies have shown the efficacy of antioxidants as therapeutic agents for ICH. It has been previously shown that the administration of dimethylthiourea, deferoxamine, or edaravone reduces brain injury in experimental models of ICH (Chen et al., 2014; Aronowski and Hall, 2005). These results suggest that compounds that interrupt the free radical cascade might improve ICH outcome. Although there are a number of ongoing clinical trials (Xi et al., 2014), currently there is no FDA-approved treatment for ICH and the use of alternative means such as green tea, which could be part of people's diet, could be an interesting, inexpensive and easy way to avoid some $\mathrm{ICH}$ sequelae without side effects. In this study, we did not show any difference on GSH levels and GPx activity. Although these are not in agreement with the literature (Shang et al.,
2013), they can be explained by differences in protocols of ICH induction and oxidative stress measurement and time.

The dose of $400 \mathrm{mg} \mathrm{kg}^{-1}$ of GT was chosen based on the study of Xu et al. (2006) in which they found memory improvement and reduced levels of pro-oxidants biomarkers after ischemic brain injury with a dose of 400 $\mathrm{mg} \mathrm{Kg} \mathrm{K}^{-1}$ but not with a dose of $100 \mathrm{mg} \mathrm{Kg}^{-1}$.

Neuroprotective interventions should be attempted before a stroke occurs (prevention) or very soon afterwards. The time to start treatment (four hours after ICH) was based on studies that revealed a therapeutic window (approximately 6 hours) between the injury onset and irreversible neuronal death related to stroke (Xu et al., 2006). ICH is a serious medical condition whose outcome can be impacted by early care (Hemphill et al., 2015). Green tea and green tea polyphenols are nontoxic, brainpermeable (Abd El Mohsen et al., 2002), natural compounds, reported to have multifunctional activities, being radical scavengers, iron chelators, anti-inflammatory and neuroprotectants as reviewed by (Mandel et al., 2004). However, the antioxidant/metal chelating activity attributed to catechin polyphenols are unlikely to be the only mechanism for their neuroprotective and neurorescue capacity. Thus, catechin polyphenols were found to invoke many mechanisms of action responsible for cellular survival (reviewed by (Weinreb et al., 2004).

\section{Conclusion}

Our study showed a neuroprotective effect of GT in an experimental model of ICH. GT attenuates neuromotor deficit and avoids the increase of ROS and lipid peroxidation. However, it is still necessary to investigate additional mechanisms that can be involved in the action of GT on this condition.

\section{Acknowledgment}

This research was funded with a student fellowship from CAPES-Brazil (Coordenação de Aperfeiçoamento de Pessoal de Nível Superior) for M.S., C.A. and A.M. and was supported by CAPES (Coordenação de Aperfeiçoamento de Pessoal de Nível Superior; PROCAD 071/2013 granted to P.M.C.) and CNPq (Conselho Nacional de Pesquisa; Edital Universal 2013 granted to P.M.C.).

\section{Author Contributions}

Mauren Assis Souza: Designed and conducted the research and had primary responsibility for the final content.

Caroline Dalla Colletta Altermann, Alexandre dos Santos Martins and Priscila Marques Sosa: Parricipated in all experiments, coordinated the dataanalysis and contributed to the writing fo the manuscript. 
Cristiano Chiapinotto Spiazzi: Conducted the research and contributed to the writing of the manuscript.

Pamela Billig Mello-Carpes: Designed the research and had primaty responsibility for the final content.

Francieli Weber Santos: Provieded essential reagents, or provided essential materials and contributed to the writing of the manuscript.

\section{Ethics}

The authors strictly observed the ethical guidelines provided in the use of the animals for this research work and ehical approval for the study was obtained from the Ethical committee for Animals Use of Federal University of Pampa. No other ethical issues are anticipated.

\section{References}

Abd El Mohsen, M.M., G. Kuhnle, A.R. Rechner, H. Schroeter and S. Rose et al., 2002. Uptake and metabolism of epicatechin and its access to the brain after oral ingestion. Free Radical Biol. Med., 33: 1693-1702. MID: 12488137

Ali, S.F., C.P. LeBel and S.C. Bondy, 1992. Reactive oxygen species formation as a biomarker of methylmercury and trimethyltin neurotoxicity. Neurotoxicology, 13: 637-648. PMID: 1475065

Aronowski, J. and C.E. Hall, 2005. New horizons for primary intracerebral hemorrhage treatment: Experience from preclinical studies. Neurol. Res., 27: 268-279. DOI: 10.1179/016164105X25225

Aronowski, J. and X. Zhao, 2011. Molecular pathophysiology of cerebral hemorrhage: Secondary brain injury. Stroke, 42: 1781-1786. PMID: 21527759

Babu, R., J.H. Bagley, M.S. Chunhui Di and C. Adamson, 2012. Thrombin and hemin as central factors in the mechanisms of intracerebral hemorrhage-induced secondary brain injury and as potential targets for intervention. Neurosurgical Focus, 32: E8-E8.

DOI: $10.3171 / 2012.1$.FOCUS11366

Bradford, M.M., 1976. A rapid and sensitive method for the quantitation of microgram quantities of protein utilizing the principle of protein-dye binding. Analytical Biochem., 72: 248-254.

DOI: 10.1016/0003-2697(76)90527-3

Chang, C.F., S. Cho and J. Wang, 2014. (-)-Epicatechin protects hemorrhagic brain via synergistic Nrf2 pathways. Ann. Clin. Trans. Neurol., 1: 258-271. DOI: 10.1002/acn3.54

Chen, Z., J. Zhang, Q. Chen, J. Guo and G. Zhu et al., 2014. Neuroprotective effects of edaravone after intraventricular hemorrhage in rats. Neuroreport, 25: 635-640. DOI: 10.1097/WNR.0000000000000050

Del Bigio, M.R., H.J. Yan, R. Buist and J. Peeling, 1996. Experimental intracerebral hemorrhage in rats: Magnetic resonance imaging and histopathological correlates. Stroke, 27: 2312-2319.

DOI: 10.1161/01.STR.27.12.2312
Flower, O. and M. Smith, 2011. The acute management of intracerebral hemorrhage. Curr. Opin. Critical Care, 17: 106-114. DOI: 10.1097/MCC.0b013e328342f823

Groenewegen, H.J., 2003. The basal ganglia and motor control. Neural Plasticity, 10: 107-120. DOI: $10.1155 /$ NP.2003.107

Hall, N.C., B.A. Packard, C.L. Hall, G. de CourtenMyers and K.R. Wagner, 2000. Protein oxidation and enzyme susceptibility in white and gray matter with in vitro oxidative stress: Relevance to brain injury from intracerebral hemorrhage. Cell. Mol. Biol., 46: 673-683. PMID: 10872754

Hemphill, J.C., S.M. Greenberg, C.S. Anderson, K. Becker and B.R. Bendok et al., 2015. Guidelines for the management of spontaneous intracerebral hemorrhage: A guideline for healthcare professionals from the American heart association/american stroke association. Stroke, 46: 2032-2060. DOI: 10.1161/STR.0000000000000069

Hissin, P.J. and R. Hilf, 1976. A fluorometric method for determination of oxidized and reduced glutathione in tissues. Analytical Biochem., 74: 214-226. PMID: 962076

Hu, X., C. Tao, Q. Gan, J. Zheng and H. Li et al., 2016. Oxidative Stress in Intracerebral Hemorrhage: Sources, Mechanisms and Therapeutic Targets. Oxidative Med. Cellular Longevity. DOI: 10.1155/2016/3215391

Hwang, B.Y., G. Appelboom, A. Ayer, C.P. Kellner and I.S. Kotchetkov et al., 2011. Advances in neuroprotective strategies: potential therapies for intracerebral hemorrhage. Cerebrovasc Dis., 31: 211-222. DOI: 10.1159/000321870

Juranek, I. and S. Bezek, 2005. Controversy of free radical hypothesis: Reactive oxygen species--cause or consequence of tissue injury? General Physiol. Biophysi., 24: 263-278. PMID: 16308423

Lu, H., J. Shen, X. Song, J. Ge and R. Cai et al., 2015. Protective effect of Pyrroloquinoline Quinone (PQQ) in Rat model of intracerebral hemorrhage. Cellular Mol. Neurobiol., 35: 921-930. PMID: 25820784

Mak, J.C., 2012. Potential role of green tea catechins in various disease therapies: Progress and promise. Clin. Experim. Pharmacol. Physiol., 39: 265-273. DOI: $10.1111 / \mathrm{j} .1440-1681.2012 .05673 . \mathrm{X}$

Mandel, S., O. Weinreb, T. Amit and M.B. Youdim, 2004. Cell signaling pathways in the neuroprotective actions of the green tea polyphenol (-)-epigallocatechin-3gallate: Implications for neurodegenerative diseases. J. Neurochem., 88: 1555-1569. PMID: 15009657

Mandel, S., T. Amit, L. Reznichenko, O. Weinreb and M.B. Youdim, 2006. Green tea catechins as brainpermeable, natural iron chelators-antioxidants for the treatment of neurodegenerative disorders. Molecular Nutrition Food Res., 50: 229-234. DOI: 10.1002/mnfr.200500156 
Ohkawa, H., N. Ohishi and K. Yagi, 1979. Assay for lipid peroxides in animal tissues by thiobarbituric acid reaction. Analytical Biochemi., 95: 351-358. DOI: 10.1016/0003-2697(79)90738-3

Paglia, D.E. and W.N. Valentine, 1967. Studies on the quantitative and qualitative characterization of erythrocyte glutathione peroxidase. J. Lab. Clin. Med., 70: 158-169. PMID: 6066618

Sadrzadeh, S.M. and J.W. Eaton, 1988. Hemoglobinmediated oxidant damage to the central nervous system requires endogenous ascorbate. J. Clin. Investigat., 82: 1510-1515. DOI: 10.1172/JCI113759

Schimidt, H.L., A. Vieira, C. Altermann, A. Martins and P. Sosa et al., 2014. Memory deficits and oxidative stress in cerebral ischemia-reperfusion: Neuroprotective role of physical exercise and green tea supplementation. Neurobiol. Learn. Memory, 114: 242-250. DOI: 10.1016/j.nlm.2014.07.005

Shah, Z.A., R.C. Li, A.S. Ahmad, T.W. Kensler and M. Yamamoto et al., 2010. The flavanol (-)-epicatechin prevents stroke damage through the Nrf2/HO1 pathway. J. Cereb. Blood Flow Metab., 30: 1951-1961. PMID: 20442725

Shang, H., D. Yang, W. Zhang, T. Li and X. Ren et al., 2013. Time course of Keap1-Nrf2 pathway expression after experimental intracerebral haemorrhage: Correlation with brain oedema and neurological deficit. Free Radical Res., 47: 368-375. DOI: 10.3109/10715762.2013.778403

Shiotsuki, H., K. Yoshimi, Y. Shimo, M. Funayama and Y. Takamatsu et al., 2010. A rotarod test for evaluation of motor skill learning. J. Neurosci. Methods, 189: 180-185. DOI: 10.1016/j.jneumeth.2010.03.026

Stroobants, S., I. Gantois, T. Pooters and R. D'Hooge, 2013. Increased gait variability in mice with small cerebellar cortex lesions and normal rotarod performance. Behav. Brain Res., 241: 32-37. PMID: 23219967

Tanabe, N., H. Suzuki, Y. Aizawa and N. Seki, 2008. Consumption of green and roasted teas and the risk of stroke incidence: Results from the TokamachiNakasato cohort study in Japan. Int. J. Epidemiol., 37: 1030-1040. PMID: 18832387

Wagner, K.R., B.A. Packard, C.L. Hall, A.G. Smulian and M.J. Linke et al., 2002. Protein oxidation and heme oxygenase- 1 induction in porcine white matter following intracerebral infusions of whole blood or plasma. Developm. Neurosci., 24: 154-160.

DOI: $10.1159 / 000065703$
Wang, J., H. Zhuang and S. Dore, 2006. Heme oxygenase 2 is neuroprotective against intracerebral hemorrhage. Neurobiol. Dis., 22: 473-476. DOI: $10.1016 /$ j.nbd.2005.12.009

Weinreb, O., S. Mandel, T. Amit and M.B. Youdim, 2004. Neurological mechanisms of green tea polyphenols in Alzheimer's and Parkinson's diseases. J. Nutrit. Biochem., 15: 506-516. PMID: 15350981

Wu, J., Y. Hua, R.F. Keep, T. Schallert and J.T. Hoff et al., 2002. Oxidative brain injury from extravasated erythrocytes after intracerebral hemorrhage. Brain Res., 953: 45-52. DOI: $10.1016 / \mathrm{S} 0006-8993(02) 03268-7$

Wu, K.J., M.T. Hsieh, C.R. Wu, W.G. Wood and Y.F. Chen, 2012. Green tea extract ameliorates learning and memory deficits in ischemic rats via its active component polyphenol epigallocatechin-3-gallate by modulation of oxidative stress and neuroinflammation. Evid. Based Complement. Alternative Med. DOI: 10.1155/2012/163106

Xi, G., J. Strahle, Y. Hua and R.F. Keep, 2014. Progress in translational research on intracerebral hemorrhage: Is there an end in sight? Progress Neurobiol., 115: 45-63. DOI: 10.1016/j.pneurobio.2013.09.007

Xi, G., R.F. Keep and J.T. Hoff, 2006. Mechanisms of brain injury after intracerebral haemorrhage. Lancet Neurol., 5: 53-63. PMID: 16361023

Xu, Y., J.J. Zhang, L. Xiong, L. Zhang and D. Sun et al., 2010. Green tea polyphenols inhibit cognitive impairment induced by chronic cerebral hypoperfusion via modulating oxidative stress. J. Nutritional Biochem., 21: 741-748. DOI: 10.1016/j.jnutbio.2009.05.002

Xu, Z., D.R. Croslan, A.E. Harris, G.D. Ford and B.D. Ford, 2006. Extended therapeutic window and functional recovery after intraarterial administration of neuregulin-1 after focal ischemic stroke. J. Cereb. Blood Flow Metab., 26: 527-535. PMID: 16136057

Yang, C.S. and J.M. Landau, 2000. Effects of tea consumption on nutrition and health. J. Nutrit., 130: 2409-2412. PMID: 11015465 